\title{
Neuroanatomical Phenotype of Klinefelter Syndrome in Childhood: A Voxel-Based Morphometry Study
}

\author{
Daniel M. Bryant, ${ }^{1,2}$ Fumiko Hoeft, ${ }^{1,2}$ Song Lai, ${ }^{3}$ John Lackey, ${ }^{3}$ David Roeltgen, ${ }^{4}$ Judith Ross, ${ }^{5,6}$ and Allan L. Reiss ${ }^{1,2,7}$ \\ ${ }^{1}$ Center for Interdisciplinary Brain Sciences Research, Stanford University School of Medicine, Stanford, California 94305-5795, ${ }^{2}$ Department of Psychiatry \\ and Behavioral Sciences, Stanford University School of Medicine, Stanford, California 94305-5795, ${ }^{3}$ Department of Radiology, Thomas Jefferson University, \\ Philadelphia, Pennsylvania 19107, ${ }^{4}$ Department of Neurology, Georgetown University, Washington, DC 20057, ${ }^{5}$ Department of Pediatrics, Thomas Jefferson \\ University, Philadelphia, Pennsylvania 19107, ${ }^{6}$ AI DuPont Hospital for Children, Wilmington, Delaware 19803, and ${ }^{7}$ Department of Radiology, Stanford \\ University School of Medicine, Stanford, California 94305-5795
}

Klinefelter syndrome (KS) is a genetic disorder characterized by a supernumerary X chromosome. As such, KS offers a naturally occurring human model for the study of both X-chromosome gene expression and androgen on brain development. Previous neuroimaging studies have revealed neuroanatomical variations associated with KS, but have differed widely with respect to subject inclusion criteria, including mosaicism, pubertal status, and history of testosterone replacement therapy (TRT), all factors likely to influence neurodevelopment. We conducted a voxel-based morphometry study of regional gray and white matter (GM and WM, respectively) volumes in 31 KS males (mean age, $9.69 \pm 1.70$ years) and 36 typically developing (TD) male controls (10.99 \pm 1.72 years). None of the participants with KS had received TRT, and all were prepubertal and had nonmosaic 47,XXY karyotypes. After controlling for age, males with KS showed trends $(0.05<p<0.10)$ for significantly reduced total gray matter volume (TGMV) and total white matter volume (TWMV), relative to TD males. After controlling for TGMV and age, the KS group had significantly increased sensorimotor and parietal-occipital GM and significantly reduced amygdalar, hippocampal, insular, temporal, and inferior frontal GM relative to TD controls. After controlling for TWMV and age, the KS group had significantly increased left parietal WM as well as significantly reduced frontal and temporal WM. These findings are indicative of a characteristic prepubertal neuroanatomical phenotype that may be associated with cognitivebehavioral features of KS. This work offers new insight into the relationships among X-chromosome gene expression, neuroanatomy, and cognitive-behavioral functions impaired in KS, including language and attention.

\section{Introduction}

Klinefelter syndrome (KS; aka 47,XXY syndrome) is a sex chromosome abnormality characterized by a supernumerary $\mathrm{X}$ chromosome. Affecting only males, KS manifests with a variety of phenotypic characteristics, including language and languagebased learning disabilities, executive and attentional dysfunction, and motor developmental delays (Geschwind et al., 2000; Wattendorf and Muenke, 2005). These characteristics have been associated with neuroanatomical variations (Itti et al., 2006). In addition, research continues to refine understanding of genetic mechanisms underlying KS (Vawter et al., 2007). The present study highlights potential downstream neuroanatomical effects of KS-related genetic differences, offering insight into the neurobehavioral phenotype associated with KS and its relevance to typical brain development.

\footnotetext{
Received Nov. 9, 2010; revised Jan. 20, 2011; accepted Feb. 24, 2011.

This study was supported by National Institutes of Health Grants R01NS050597 (to J.R.) and R01HD049653 (to A.L.R.). We acknowledge the support of the families involved in the study. We thank the Department of Radiology, Thomas Jefferson University for providing the MRI scanner time via a research support initiative to S.L. We thank Jianrong Shi for her technical support in the MRI data acquisition. We thank Dr. Amy Garrett for her imaging support.

Correspondence should be addressed to Dr. Allan L. Reiss, Center for Interdisciplinary Brain Sciences Research, MC 5795, Department of Psychiatry and Behavioral Sciences, Stanford University School of Medicine, Stanford, CA 94305. E-mail: areiss1@stanford.edu.

DOI:10.1523/JNEUROSCI.5899-10.2011

Copyright $\odot 2011$ the authors $\quad 0270-6474 / 11 / 316654-07 \$ 15.00 / 0$
}

Structural imaging studies have revealed significant neuroanatomical differences associated with KS. Many studies report reduced total brain volume (TBV) in KS (Warwick et al., 1999; Shen et al., 2004; DeLisi et al., 2005), though some report no significant difference (Patwardhan et al., 2002; Itti et al., 2006). In addition, KS males typically have reduced frontal and temporal gray matter (GM) (Shen et al., 2004; DeLisi et al., 2005). Some studies report differences in white matter (WM) (Rezaie et al., 2009), whereas others do not (Giedd et al., 2007). Discrepant findings may result from variation in subject age, sample size, neuroimaging acquisition techniques, and neuroimaging analysis methods (Steinman et al., 2009). Most notably, structural neuroimaging studies of KS vary with regard to inclusion of participants who have begun puberty or received testosterone replacement therapy (TRT), both of which are likely to influence brain development (Patwardhan et al., 2000; Reiss et al., 2000; Lanfranco et al., 2004; Wattendorf and Muenke, 2005).

We conducted a rigorous analysis of structural brain differences in KS by minimizing the influence of puberty-associated androgen effects on brain development. Specifically, we excluded potential KS participants if they had begun puberty, had undergone TRT, or showed evidence of chromosomal mosaicism. Though we could not eliminate the influence of early developmental differences, including varied androgen levels throughout childhood (Ross et al., 2005), we hoped to capture the neuroana- 
Table 1. Demographic information and cognitive testing

\begin{tabular}{|c|c|c|c|c|c|c|}
\hline \multirow[b]{2}{*}{ Characteristic } & \multirow[b]{2}{*}{ N } & \multicolumn{2}{|l|}{ Mean (SD) } & \multirow[b]{2}{*}{$t$ value } & \multirow[b]{2}{*}{$\chi^{2}$} & \multirow[b]{2}{*}{$p$ value } \\
\hline & & KS & TD & & & \\
\hline Age (years) & $31 \mathrm{KS}, 36 \mathrm{TD}$ & $9.69(1.70)$ & $10.99(1.72)$ & -3.104 & - & 0.003 \\
\hline DAS, nonverbal cluster & $30 \mathrm{KS}, 33 \mathrm{TD}$ & $96.47(15.76)$ & $111.15(13.68)$ & -3.959 & - & $<0.001$ \\
\hline DAS, verbal cluster & $30 \mathrm{KS}, 33 \mathrm{TD}$ & $88.80(14.15)$ & $112.70(14.50)$ & -6.608 & - & $<0.001$ \\
\hline DAS, spatial cluster & 30 KS, 34 TD & 92.33 (15.22) & $104.65(14.84)$ & -3.273 & - & 0.002 \\
\hline
\end{tabular}

Numbers in parentheses represent \pm SD. ambi., Ambidextrous.

tomical phenotype of KS during a critical developmental window, making possible direct comparison with other, mostly adult studies of $\mathrm{KS}$ and offering insight into neuroanatomical variations more closely associated with the 47,XXY genotype.

We hypothesized that KS males would have significantly reduced total gray matter volume (TGMV) and total white matter volume (TWMV), consistent with a previous voxel-based morphometry (VBM) study (Shen et al., 2004). We also predicted abnormalities in regions associated with language and verbal memory [including left frontal and temporal areas (Geschwind, 1979) and insular cortex (Wise et al., 1999)], sensorimotor function [including precentral and postcentral gyri (Penfield and Boldrey, 1937), basal ganglia (Evarts, 1973), and corpus callosum (for bimanual motor function) (Jeeves et al., 1988)], and memory retrieval and working memory [including hippocampus (Cohen and Eichenbaum, 1993), parietal lobe (Bledowski et al., 2009), and prefrontal cortex (Haxby et al., 2000)]. Finally, we expected abnormalities in amygdalar and cingulate regions because of mood and behavior dysregulation reported in KS males (Mandoki et al., 1991; Bender et al., 1995).

\section{Materials and Methods}

Participants. All participants were male, as KS only affects males. Participants with KS were recruited from the Thomas Jefferson University pediatric endocrine clinic (Philadelphia, PA), were self-referred, or were referred by other physicians. Control subjects were recruited by internet notices and by referrals from other families in research studies. Both KS and typically developing (TD) participants were recruited at 7-14 years of age and the groups were matched for prepubertal status. Pubertal status was determined using standard clinical methods assessing testicular volume (Marshall and Tanner, 1970). Participants with a history of TRT were excluded from the analysis. Endogenous testosterone levels were also measured in the KS group, but not in TD controls. Compared with well known averages for TD boys of this age, all but five of the boys with KS had testosterone levels similar to those of prepubertal typically developing males, as expected. Five boys with KS had testosterone levels within the early pubertal range, but they did not meet the testicularvolume-dependent criterion for puberty onset, and so were included in the sample. However, to assess the possibility that their inclusion biased the results, separate analyses were performed on a KS subsample that did not include these subjects, as described below.

The KS diagnosis $(47, \mathrm{XXY})$ was confirmed by karyotype in each participant. A postnatal, G-banded, peripheral blood karyotype was obtained for all subjects. Each karyotype included $\geq 20$ cells. Two participants had low levels ( $<20 \%)$ of mosaicism for a 46,XY cell line and were excluded. The Institutional Review Boards at Thomas Jefferson University and Stanford University approved the study. Informed consent was obtained from all parents and assent was obtained from all participants.

Subjects were given a standard battery of psychological measures including the Crovitz-Zener test for measuring handedness (Crovitz and Zener, 1962) and the Differential Ability Scales (DAS) for verbal, nonverbal, and spatial abilities (Elliott, 1990). Table 1 reports details and between-group statistics.
Of the 94 participants for whom structural MRI data were available, 67 participants ( $36 \mathrm{TD}, 31 \mathrm{KS}$ ) were included in the analyses. Twentytwo scans were excluded (5 TD, $17 \mathrm{KS}$ ) due to excessive in-scanner motion, as determined visually by an experienced investigator who was blind to diagnosis (F.H.). Five additional KS subjects were eliminated in accordance with exclusion criteria (two for prior TRT, two for mosaicism, one for XXYY karyotype). To assess the effects of the five boys with KS with higher testosterone levels, a secondary analysis was conducted without these subjects, resulting in a 62-participant subsample (36 TD, $26 \mathrm{KS}$ ). A third confirmatory analysis was also conducted that included an age-matched subsample of 54 participants (27 TD, $27 \mathrm{KS}$ ). To create this subsample, 13 scans (9 TD, $4 \mathrm{KS}$ ) were excluded from the main sample. Age matching was achieved by eliminating the oldest TD participants and youngest participants with KS until the between-group $t$ test of age yielded a significance value ( $p$ value) $>0.4$, indicating no significant age difference or trend (Table 1 ).

MRI data acquisition. All MRI scanning was performed on a Philips 3.0T whole-body clinical MRI system (Achieva; Philips Medical Systems) equipped with a Quasar Dual high-performance gradient system capable of on-axis $(x, y$, and $z)$ peak gradient of $80 \mathrm{mT} / \mathrm{m}$ and $200 \mathrm{mT} / \mathrm{m} / \mathrm{ms}$ slew rate, and an eight-channel SENSE (sensitivity encoding) head coil.

Structural images were obtained using a conventional, highresolution, three-dimensional $\mathrm{T} 1$-weighted fast gradient echo sequence [repetition time, $25 \mathrm{~ms}$; echo time, $2.3 \mathrm{~ms}$; angle, $30^{\circ}$; $0.96 \times 0.96 \times 1$ $\mathrm{mm}^{3}$ voxels; 160 contiguous anterior commissure-posterior commissure (AC-PC)-aligned slices of $1 \mathrm{~mm}$ thickness; acquisition time, $6 \mathrm{~min}$ and $9 \mathrm{~s}]$.

MRI data analysis. VBM analysis of T1-weighted MRIs was performed using Statistical Parametric Mapping, version 8 (SPM8; http://www.fil. ion.ucl.ac.uk/spm). After image conversion to Nifti format using r2agui (http://r2agui.sourceforge.net/) and alignment to AC-PC axis, T1weighted images were bias-corrected and segmented to GM, WM, and CSF, using SPM8 default tissue probability maps and the New Segment tool, which also included an affine regularization to warp images to the included International Consortium for Brain Mapping template, producing rigidly aligned tissue class images. Intersubject registration was achieved with Diffeomorphic Anatomical Registration Through Exponentiated Lie Algebra (DARTEL), using default settings. Jacobian-scaled (modulated) warped tissue class images were created with DARTEL's Normalize to MNI Space tool, which spatially normalized images to MNI space, converted voxel sizes to $1.5 \times 1.5 \times 1.5 \mathrm{~mm}^{3}$ to match the DARTEL template, and smoothed images with a standard Gaussian filter of full-width at half-maximum equal to $8 \mathrm{~mm}$.

Regional GM and WM volumes were compared between KS and TD groups with two-sample $t$ tests in SPM, using age and appropriate uncorrected brain matter volume (TGMV or TWMV) as covariates. Table 2 reports total gray and white matter volumes after correction for age. The search region was defined with an explicit GM or WM mask, created by averaging the unsmoothed, modulated, warped tissue class images. Four between-group contrasts were defined (GM, KS $>$ TD; GM, KS $<\mathrm{TD}$; $\mathrm{WM}, \mathrm{KS}>\mathrm{TD}$; WM, KS $<\mathrm{TD}$ ). Analyses were performed using a height threshold of $p<0.01$ and an extent threshold of $p<0.01$, corrected for family-wise error (FWE). All comparisons were corrected for nonstationarity smoothness (Hayasaka et al., 2004) with VBM5.1 toolbox (http://dbm.neuro.uni-jena.de/vbm). To assess the relationship between 
cognitive ability, testosterone levels, and neuroanatomical differences, whole-brain correlations with cognitive-behavioral scores and testosterone measures were performed using multiple regression in SPM. Age and appropriate brain matter volume (TGMV or TWMV) were included as nuisance covariates. Analyses were performed using an FWE-corrected height threshold of $p<0.05$ and an extent threshold of $k>20$. Resultant contrasts were masked using small-volume correction to include only those areas showing between-group brain matter differences in the primary analysis. Final results were visualized with MRIcron (http://www. cabiatl.com/mricro) and xjView (http://www.alivelearn.net/xjview). Gray and white matter (GM/WM) peak voxel region labels were assigned with Talairach Daemon (Lancaster et al., 1997, Lancaster et al., 2000) after coordinate conversion, and were visually confirmed with the Co-Planar Stereotaxic Atlas of the Human Brain (Talairach and Tornoux, 1988).

\section{Results}

KS and TD groups showed significantly different verbal and nonverbal ability, as measured by the DAS verbal, nonverbal, and spatial clusters. Whole-brain analysis showed that KS males had significantly reduced TGMV and TWMV compared with TD participants. This became a trend for significance after correction for age (Table 2). After controlling for TGMV and age, VBM analyses showed that participants with KS had disproportionately larger GM in bilateral precentral and postcentral gyri as well as in bilateral parietal-occipital regions, extending into the occipital pole and including bilateral precunei, cunei, superior parietal lobules, and bilateral calcarine sulci (Fig. 1, Table 3 ). The KS group showed significantly reduced GM in the bilateral insulae, bilateral inferior frontal gyri, bilateral amygdalae, right middle gyri, superior temporal gyri (STG), bilateral parahippocampal cortices, and bilateral hippocampi (Fig. 1, Table 3).

In contrast, WM comparisons revealed significantly increased WM in KS in the left parietal region, extending into the occipital lobe, after controlling for TWMV and age. Participants with KS also had significantly reduced WM relative to TD males in frontal and temporal lobes, including areas near bilateral orbitofrontal cortex (OFC), bilateral subgenual, and bilateral superior temporal sulcus (STS) (Fig. 2, Table 4).

Whole-brain multiple regression analysis of the relationship between cognitivebehavioral measures or testosterone level and GM or WM volume did not yield statistically significant results within KS. In the age-matched subsample, the results were highly similar (same cluster locations and significance level), though slightly more robust. In the subsample without high-testosterone participants with KS, contrasts were also highly similar to those of the primary sample.
Table 2. Brain matter volumes in KS and TD groups, corrected for age

\begin{tabular}{|c|c|c|c|c|}
\hline & \multicolumn{2}{|l|}{ Mean (SD) } & \multirow[b]{2}{*}{$t$ value } & \multirow[b]{2}{*}{$p$ value } \\
\hline & KS & TD & & \\
\hline GMV & $717.66(65.45)$ & $744.63(55.37)$ & -1.827 & 0.072 \\
\hline TWMV & $485.23(45.76)$ & 505.94 (42.56) & -1.919 & 0.059 \\
\hline TGMV + TWMV & $1202.88(110.06)$ & 1250.57 (94.96) & -1.904 & 0.061 \\
\hline
\end{tabular}

Numbers in parentheses represent \pm SD.

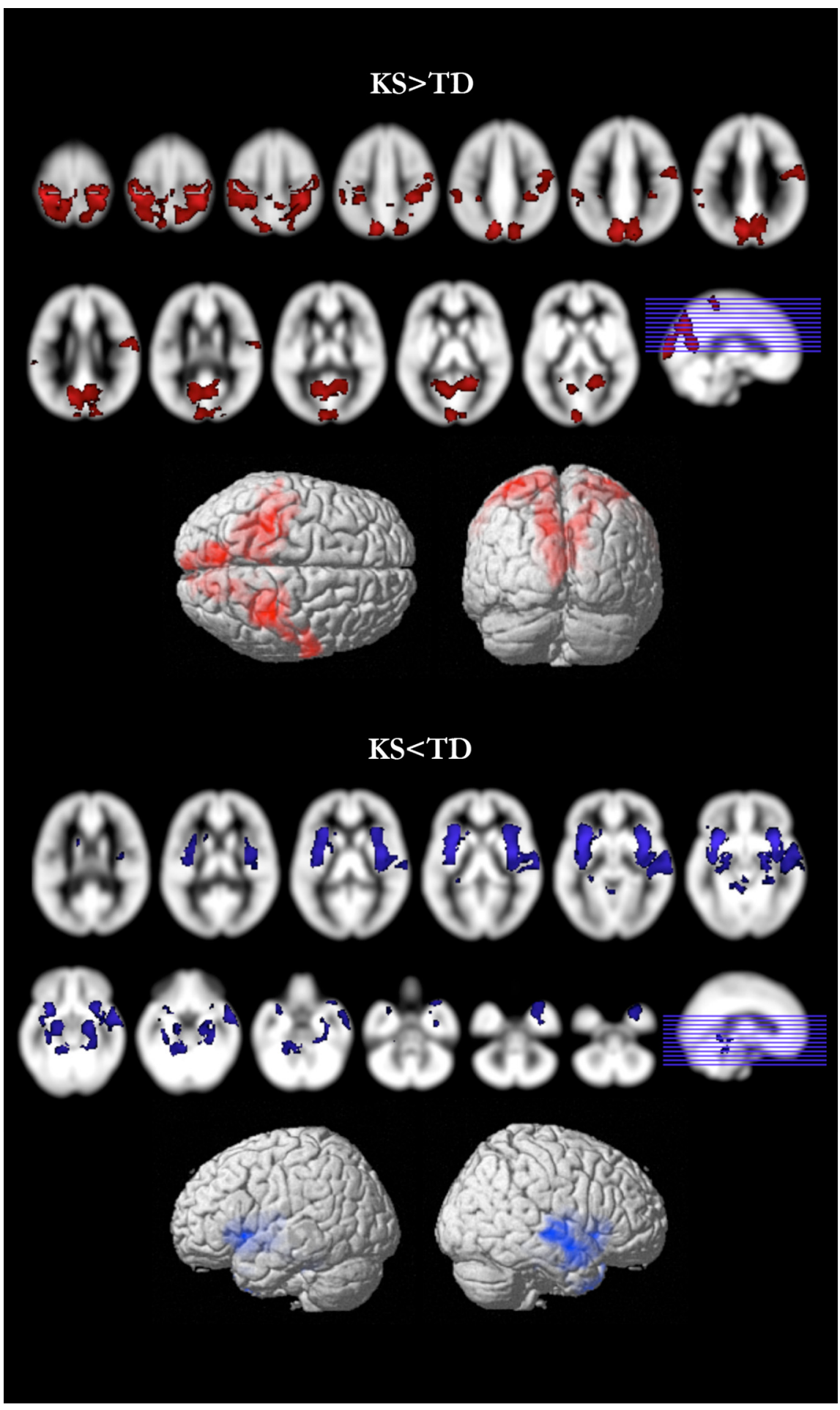

Figure 1. Gray matter differences. Regional GM differences between KS and TD groups. Contrasts with significant differences $(p<0.01$, corrected) are overlaid on the SPM8 a priori GM template. KS $>$ TD is shown in red; $K S<$ TD is shown in blue. 
Table 3. GM regions showing significant difference between $\mathrm{KS}$ and TD groups

\begin{tabular}{|c|c|c|c|c|c|}
\hline Region & $\begin{array}{l}\text { Brodmann } \\
\text { area }\end{array}$ & $\begin{array}{l}\text { Peak voxel MNI } \\
\text { coordinates }\end{array}$ & $\begin{array}{l}t \text { value, } \\
\text { voxel-level }\end{array}$ & $\begin{array}{l}p \text { value } \\
\text { (FWE) }\end{array}$ & $\begin{array}{l}\text { Cluster } \\
\text { size }^{a}\end{array}$ \\
\hline \multicolumn{6}{|l|}{$\mathrm{KS}>\mathrm{TD}$} \\
\hline L postcentral gyrus & 1 & $-33,-35$ & 667.69 & & \\
\hline R postcentral gyrus & 5 & $34,-38$ & 667.10 & $<0.001$ & 38,685 \\
\hline L postcentral gyrus & 3 & $-26,-39$ & 726.62 & & \\
\hline \multicolumn{6}{|l|}{$\mathrm{KS}<\mathrm{TD}$} \\
\hline L anterior insula & 13 & $-34,9$ & -107.14 & & \\
\hline L superior temporal sulcus & 41 & $-38,-9$ & -74.85 & $<0.001$ & 7189 \\
\hline L superior temporal sulcus & 41 & $-44,0$ & -74.25 & & \\
\hline R anterior insula & 13 & 39,10 & -96.84 & & \\
\hline R insula & 13 & $40,-2$ & 26.27 & $<0.001$ & 10,959 \\
\hline R superior temporal gyrus & 22 & $66,-15$ & -15.14 & & \\
\hline
\end{tabular}

Regions of interest are listed for peak voxels and subpeaks only. See Results and Figure 1 for additional regions of GM difference. L, Left; R, right.

${ }^{a}$ Voxel size: $1.5 \times 1.5 \times 1.5 \mathrm{~mm}$

GM in bilateral insulae, bilateral inferior frontal gyri, bilateral temporal lobes, bilateral amygdalae, and bilateral hippocampi. WM contrasts revealed that the KS group had significantly increased leftward parietal and occipital WM after controlling for age and TWMV as well as significantly reduced WM in frontal and temporal regions, including areas near bilateral OFC, bilateral subgenual, bilateral STS, and right STG. These morphological differences may represent a characteristic neuroanatomical phenotype of prepubertal boys with KS who are TRT-naive. In addition, these differences may represent the downstream neuroanatomical correlates of abnormal X-chromosome gene expression associated with KS.

The findings reported here support many of our a priori hypotheses, but introduce a more complicated picture of KS neuroanatomy than expected. For example, we anticipated neuroanatomical differences in precentral and postcentral gyri, as individuals with KS commonly exhibit sensorimotor impairments.

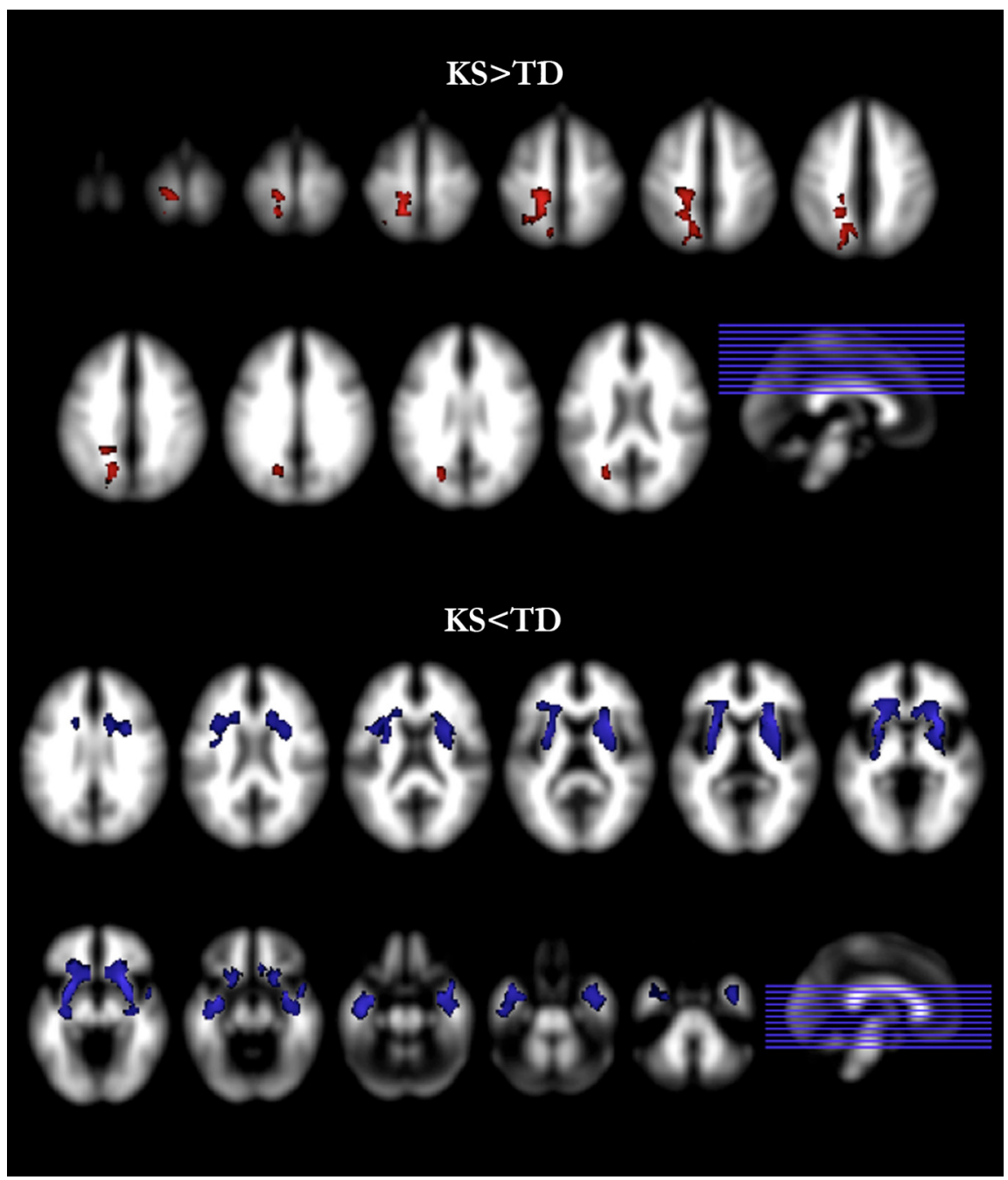

Figure 2. White matter differences. Regional WM differences between KS and TD groups. Contrasts with significant differences $(p<0.01$, corrected) are overlaid on the SPM8 a priori WM template. KS $>$ TD is shown in red; KS $<$ TD is shown in blue. However, we did not predict GM increase in these regions or in other areas of the parietal and occipital cortices. To our knowledge, relatively increased parietal-occipital GM has not been reported previously in KS. This finding suggests a variety of interpretations. For example, observed GM increases in sensorimotor and occipital regions in KS could actually reflect relative sparing in these areas combined with global correction for TGMV. Though there was still a trend for significant difference in TGMV between KS and TD after controlling for age, this difference might not have arisen from uniformly distributed GM reduction in KS. Thus, whole-brain correction for TGMV may have emphasized regions not contributing to TGMV reduction, chiefly, the sensorimotor and occipital regions. A study by Giedd et al. (2007) may support this interpretation, as it shows relative sparing of parietal GM compared with frontal and temporal GM in KS.

In contrast, increased GM in sensorimotor and occipital regions in KS could result from reduced synaptic maturation or pruning, leading to an increased volume of neuropil, diminished synaptic efficiency, and associated sensorimotor deficits in affected individuals. Given that we identified reduced GM in other regions of the KS brain, the plausibility of such a hypothesis requires speculation that downstream neurobiological changes resulting from altered $\mathrm{X}$-chromosome gene expression in $\mathrm{KS}$ have temporally specific effects on differing brain regions. Though region-specific critical windows of development resulting

\section{Discussion}

Structural comparisons revealed total and regional GM and WM differences between KS and TD males. VBM analysis showed that boys with KS had disproportionately increased GM in parietal and occipital cortices, including sensorimotor areas, after controlling for TGMV and age. Boys with KS showed disproportionately decreased in increased and decreased volumes have been described in other conditions such as fragile X syndrome (Hoeft et al., 2010), the issue clearly warrants further investigation in KS using prospective, longitudinal assessment methods. We plan to directly assess the influence of TRT on volumetric differences in parietal GM and other areas of the brain in a 2 year follow-up study. 
Table 4. WM regions showing significant difference between $\mathrm{KS}$ and TD groups

\begin{tabular}{|c|c|c|c|c|}
\hline Nearby regions & $\begin{array}{l}\text { Peak voxel MNI } \\
\text { coordinates }\end{array}$ & $\begin{array}{l}t \text { value } \\
\text { (voxel-level) }\end{array}$ & $\begin{array}{l}p \text { value, } \\
\text { cluster-level, } \\
\text { nonstationarity } \\
\text { adjusted }\end{array}$ & $\begin{array}{l}\text { Cluster } \\
\text { size }^{a}\end{array}$ \\
\hline \multicolumn{5}{|l|}{$\mathrm{KS}>\mathrm{TD}$} \\
\hline L parietal lobe, precuneus & $-15,-44$ & $60 \quad 6.26$ & & \\
\hline Loccipital lobe, cuneus & $-22,-68$ & $23 \quad 4.65$ & 0.003 & 4815 \\
\hline L parietal lobe, precuneus & $-20,-65$ & $42 \quad 4.41$ & & \\
\hline \multicolumn{5}{|l|}{$\mathrm{KS}<\mathrm{TD}$} \\
\hline R claustrum & 27,18 & $\begin{array}{ll}-9 & 7.57\end{array}$ & & \\
\hline R claustrum & 31,4 & $-15 \quad 5.93$ & 0.003 & 4614 \\
\hline R claustrum & 30,10 & 85.75 & & \\
\hline L amygdala, extended & $-33,0$ & $-18 \quad 6.46$ & & \\
\hline L claustrum & $-28,10$ & $-10 \quad 6.25$ & $\{0.002$ & 5102 \\
\hline L middle temporal lobe & $-42,-14$ & $-21 \quad 5.55$ & & \\
\hline
\end{tabular}

Regions of interest are listed for peak voxels and subpeaks only. See Results and Figure 2 for additional regions of WM difference. L, Left; $R$, right.

${ }^{a}$ Voxel size: $1.5 \times 1.5 \times 1.5 \mathrm{~mm}$

The proposed longitudinal assessment may offer a better understanding of the observed increase in parietal GM. For example, if TRT does not influence parietal GM differences, or if TRT is associated with a further increase in parietal GM, one might interpret this to suggest that the observed increase results from GM sparing. In contrast, if TRT mitigates or eliminates the observed parietal GM increase, the latter explanation, that of reduced synaptic maturation, may be more likely. This latter explanation may also partly account for why adult studies of KS do not report increased parietal GM; if such an increase results from androgen deficiency, testosterone increase related to puberty or TRT might mitigate this adverse neuroanatomical effect. Of course, several other factors likely contribute to the discrepancy between the results presented here and those of other studies, as noted in the Introduction and later in the Discussion. Nonetheless, the specificity of the developmental window assessed in this study makes possible the comparison of pre-TRT and post-TRT neuroanatomical profiles in KS, allowing direct assessment of hormonal influences on neuroanatomy and offering insight into developmental trajectories in normal and abnormal sexual dimorphism.

Relative increase in regional GM in KS also suggests a putative relationship between parietal lobe development and $\mathrm{X}$-chromosome gene dosage, especially when considered alongside neuroanatomical variations associated with Turner syndrome (TS). TS is a chromosomal aneuploidy syndrome resulting from the complete or partial absence of one X chromosome in females. Accordingly, whereas the TS phenotype results from reduced dosage of selected $\mathrm{X}$-chromosome genes relative to typically developing individuals, the KS phenotype is associated with increased dosage of these same X-chromosome genes. Thus, the contrast between neuroanatomical differences in TS and KS relative to sex- and age-matched, typically developing children offers a compelling model for the study of X-chromosome gene effects on brain development. For example, females with TS have bilateral parietal lobe reduction, specifically in the superior parietal and postcentral gyri (Brown et al., 2004), and increased volume of the superior and middle temporal cortices (Kesler et al., 2003; Rae et al., 2004). Therefore, girls with TS have parietal and temporal lobe differences that are both overlapping with and in the opposite direction of neuroanatomical variations found in boys with KS. This suggests an X-chromosome gene dosage effect on parietal and temporal lobe development, though the suggested relationship is more complex than presented here, given that the observed neuroanatomical profiles of each condition result from the complicated interplay of X-chromosome gene dosage differences and a variety of other postnatal influences, including the effects of an altered neuroendocrine milieu and environmental factors.

Boys with KS in the present study also had increased left parietal WM, which may be associated with working memory (Takeuchi et al., 2010). However, as with the increase in parietal GM, the observed increase in left parietal WM may result from relative sparing in this area combined with correction for TWMV. Another VBM study (Shen et al., 2004) may support this interpretation, showing reduced right parietal WM in KS, which may reflect a similar pattern of neuroanatomical differences, but one resulting from analyses without direct correction for TWMV. In addition, a third study (Giedd et al., 2007) reported increased parietal WM in KS after correction for total cerebral volume, but no differences in parietal WM before correction. Differences among these studies and the present study likely stem from variations in neuroimaging analysis methods, but may also derive from sample age and developmental status.

The present study also reveals a distinct picture of KS neuroanatomy in areas related to language function. Specifically, boys with KS showed disproportionately reduced GM in languageassociated areas, which we predicted based on reports of language impairment in KS (Graham et al., 1988; Geschwind et al., 2000) and our KS group's significantly reduced verbal ability (Table 1). However, we expected primarily left-lateralized GM reductions in boys with KS, given the typical localization of speech function in the left hemisphere in right-handed participants (Geschwind and Levitsky, 1968; Binder et al., 1997). In addition, a previous VBM study of KS (Shen et al., 2004) reported primarily leftlateralized GM reduction in insular and temporal regions as well as bilateral GM reduction in the STG. In contrast, we found bilateral insular, temporal, and frontal GM reduction as well as right-lateralized GM reduction in the STG. These findings are similar to those of DeLisi et al. (2005), who reported smaller bilateral frontal and temporal lobe volumes, including bilateral STG volumes, in males with KS. Though language can elicit bilateral activation of temporal lobes (Lehéricy et al., 2000), it is unclear whether reduced right temporal GM in KS is related to impaired language ability. Brain matter differences in these rightsided language homologues may also underlie impairments in attention and social cognition, which have been shown to be associated with these regions (Puce et al., 1998; Redcay et al., 2010) and are often impaired in KS (Geschwind et al., 2000; van Rijn et al., 2006). Nonetheless, it is possible that the observed GM differences in these regions do indeed underlie aspects of language dysfunction, as individuals with KS show increased rightsided brain activity during language processing tasks, relative to TD controls (van Rijn et al., 2008). This may also be related to the higher incidence of left-handedness, commonly associated with abnormal cerebral lateralization, found in KS (Ross et al., 2008). We plan to further address questions related to the brain basis of language dysfunction in KS in future functional MRI studies of verbal processing.

WM reductions in boys with KS observed here might also be associated with language abnormalities in KS. In particular, the existence of WM reductions in frontal and temporal regions near OFC, STS, and right STG suggest an abnormal connectivity among a variety of frontal and temporal areas known to function together in human language (Matsumoto et al., 2004). Overall, GM and WM reductions within frontal and temporal regions provide a plausible neuroanatomical profile that may underlie 
commonly observed cognitive-behavioral deficits in KS, including impairments in language, attention, and executive function, which often manifest simultaneously and influence one another.

In the present study, regional GM reductions in hippocampus, parahippocampal cortices, and amygdala in boys with KS support our initial hypotheses and suggest a putative neuroanatomical substrate for memory impairment and mood dysregulation in KS. Specifically, reductions in these regions may underlie increased incidence of psychiatric disorders (Mandoki et al., 1991; Bender et al., 1995). In addition, amygdalar reductions may be related to the atypical temperament, passivity, and reduced sexual drive associated with KS (Patwardhan et al., 2002).

In contrast to the present study, some previous investigations have reported no structural differences in the amygdala-hippocampal complex (Warwick et al., 1999; DeLisi et al., 2005) or the hippocampal complex alone (Itti et al., 2006), whereas others have not reported findings pertaining to the hippocampus or amygdala (Giedd et al., 2007). One study (Patwardhan et al., 2002) found reductions in amygdalar volume, but not in hippocampal volume. In contrast, two studies (Rose et al., 2004; Shen et al., 2004) found reductions in amygdalar and hippocampal volumes, but did not correct for TBV by traditional means. Accordingly, methodological factors may underlie discrepancies in measures of amygdalar and hippocampal differences.

Observed amygdalar and hippocampal reductions might also relate to the developmental status of the participants. Interestingly, the studies reporting no significant differences in amygdala-hippocampal volumes (Warwick et al., 1999; DeLisi et al., 2005) or hippocampal volumes (Patwardhan et al., 2002; Itti et al., 2006) included all adult subjects, though Patwardhan et al. (2002) did report amygdalar differences in an all-adult sample. Nonetheless, it is tempting to speculate that testosterone increase, related either to puberty or TRT, might mitigate KS-related volumetric reductions in the amygdala, hippocampus, or both, especially given the highly sexually dimorphic nature of these regions (Giedd et al., 1996) and evidence of androgen receptor localization in the hippocampus (Gould et al., 1990) and amygdala (Simerly et al., 1990), specifically in the medial posterior dorsal nucleus (Cooke et al., 1999). Whatever their underlying cause, the amygdalar and hippocampal reductions observed in the present study and their potential relation to the developmental status of the subject sample illustrate the importance of studying KS in young populations.

Appropriate interpretation of the present findings requires an understanding of the variable developmental trajectories observed in individuals with KS. For example, testosterone deficiencies have been reported in infants with KS, and some boys with KS maintain low levels of testosterone throughout childhood (Topper et al., 1982; Ross et al., 2005). In addition, some individuals with KS do not experience pubertal testicular enlargement, whereas others experience varying degrees of enlargement (Ratcliffe et al., 1982). This variability complicates definition of specific pubertal milestones in KS. For example, reliance on Tanner criteria to define the onset and effects of spontaneous, endogenous hormonal influences on brain development in KS may not be accurate in all affected individuals.

Two potential limitations of this study involve measurement of testosterone levels in the KS sample. First, we were only able to measure testosterone levels once, at each subject's visit, so we cannot rule out the possibility that prenatal and postnatal endogenous testosterone fluctuations contributed to observed neuroanatomical changes. Second, our overall sample included five boys who had testosterone levels in the early pubertal range, de- spite their meeting the testicular size criterion for prepubertal status. To assess the possibility that their inclusion might have biased the results, we created a subsample with these five outliers excluded. Results from the analysis of this subsample were very similar to those reported for the entire group, so we believe that the inclusion of the five boys did not inordinately bias results, though we cannot eliminate the possibility. Finally, though we believe that our inclusion criteria-prepubertal and without history of TRT-improved our ability to disentangle downstream effects of the KS genotype from potentially confounding variables, it is important to note that these criteria are themselves inextricably interwoven with a variety of factors including age, hormonal status, and severity of the condition.

The results of this study represent a robust, whole-brain quantification of neurodevelopmental alterations associated with KS without the confounding influences of exogenous testosterone replacement; spontaneous, outwardly manifest onset of puberty; and karyotypic mosaicism. More generally, this research offers insight into the effects of a supernumerary X chromosome and androgen on structural brain development, suggesting a relationship with language lateralization and ability. In the future, we plan to apply similar neuroimaging approaches to directly assess the potential benefit of androgen replacement therapy in boys with this common disorder. Continued research of this nature is important both for its direct benefit to boys with KS and for its ability to elucidate genetic and hormonal influences on cognition, behavior, and brain anatomy.

\section{References}

Bender BG, Harmon RJ, Linden MG, Robinson A (1995) Psychosocial adaptation of 39 adolescents with sex chromosome abnormalities. Pediatrics 96:302-308.

Binder JR, Frost JA, Hammeke TA, Cox RW, Rao SM, Prieto T (1997) Human brain language areas identified by functional magnetic resonance imaging. J Neurosci 17:353-362.

Bledowski C, Rahm B, Rowe JB (2009) What "works" in working memory? Separate systems for selection and updating of critical information. JNeurosci 29:13735-13741.

Brown WE, Kesler SR, Eliez S, Warsofsky IS, Haberecht M, Reiss AL (2004) A volumetric study of parietal lobe subregions in Turner syndrome. Dev Med Child Neurol 46:607-609.

Cohen NJ, Eichenbaum H (1993) Memory, amnesia, and the hippocampal system. Cambridge: MIT.

Cooke BM, Tabibnia G, Breedlove SM (1999) A brain sexual dimorphism controlled by adult circulating androgens. Proc Natl Acad Sci U S A 96:7538-7540.

Crovitz HF, Zener K (1962) A group-test for assessing hand- and eyedominance. Am J Psychol 75:271-276.

DeLisi LE, Maurizio AM, Svetina C, Ardekani B, Szulc K, Nierenberg J, Leonard J, Harvey PD (2005) Klinefelter's syndrome (XXY) as a genetic model for psychotic disorders. Am J Med Genet B Neuropsychiatr Genet 135B:15-23.

Elliott CD (1990) Differential abilities scale. New York: Psychological Corporation.

Evarts EV (1973) Brain mechanisms in movement. Sci Am 229:96-103.

Geschwind DH, Boone KB, Miller BL, Swerdloff RS (2000) Neurobehavioral phenotype of Klinefelter syndrome. Ment Retard Dev Disabil Res Rev 6:107-116.

Geschwind N (1979) Specializations of the human brain. Sci Am 241:180-199.

Geschwind N, Levitsky W (1968) Human brain: left-right asymmetries in temporal speech region. Science 161:186-187.

Giedd JN, Vaituzis AC, Hamburger SD, Lange N, Rajapakse JC, Kaysen D, Vauss YC, Rapoport JL (1996) Quantitative MRI of the temporal lobe, amygdala, and hippocampus in normal human development: ages 4-18 years. J Comp Neurol 366:223-230.

Giedd JN, Clasen LS, Wallace GL, Lenroot RK, Lerch JP, Wells EM, Blumenthal JD, Nelson JE, Tossell JW, Stayer C, Evans AC, Samango-Sprouse CA 
(2007) XXY (Klinefelter syndrome): a pediatric quantitative brain magnetic resonance imaging case-control study. Pediatrics 119:e232-e240.

Gould E, Woolley CS, Frankfurt M, McEwen BS (1990) Gonadal steroids regulate dendritic spine density in hippocampal pyramidal cells in adulthood. J Neurosci 10:1286-1291.

Graham JM Jr, Bashir AS, Stark RE, Silbert A, Walzer S (1988) Oral and written language abilities of XXY boys: implications for anticipatory guidance. Pediatrics 81:795-806.

Haxby JV, Petit L, Ungerleider LG, Courtney SM (2000) Distinguishing the functional roles of multiple regions in distributed neural systems for visual working memory. Neuroimage 11:380-391.

Hayasaka S, Phan KL, Liberzon I, Worsley KJ, Nichols TE (2004) Nonstationary cluster-size inference with random field and permutation methods. Neuroimage 22:676-687.

Hoeft F, Carter JC, Lightbody AA, Cody Hazlett H, Piven J, Reiss AL (2010) Region-specific alterations in brain development in one- to three-year-old boys with fragile X syndrome. Proc Natl Acad Sci U S A 107:9335-9339.

Itti E, Gaw Gonzalo IY, Pawlikowska-Haddal A, Boone KB, Mlikotic A, Itti L, Mishkin FS, Swerdloff RS (2006) The structural brain correlates of cognitive deficits in adults with Klinefelter's syndrome. J Clin Endocrinol Metab 91:1423-1427.

Jeeves MA, Silver PH, Milne AB (1988) Role of the corpus callosum in the development of bimanual motor skill. Dev Neuropsychol 4:305-323.

Kesler SR, Blasey CM, Brown WE, Yankowitz J, Zeng SM, Bender BG, Reiss AL (2003) Effects of X-monosomy and X-linked imprinting on superior temporal gyrus morphology in Turner syndrome. Biol Psychiatry 54:636-646.

Lancaster JL, Rainey LH, Summerlin JL, Freitas CS, Fox PT, Evans AC, Toga AW, Mazziotta JC (1997) Automated labeling of the human brain: a preliminary report on the development and evaluation of a forwardtransform method. Hum Brain Mapp 5:238-242.

Lancaster JL, Woldorff MG, Parsons LM, Liotti M, Freitas CS, Rainey L, Kochunov PV, Nickerson D, Mikiten SA, Fox PT (2000) Automated Talairach atlas labels for functional brain mapping. Hum Brain Mapp 10:120-131.

Lanfranco F, Kamischke A, Zitzmann M, Nieschlag E (2004) Klinefelter's syndrome. Lancet 364:273-283.

Lehéricy S, Cohen L, Bazin B, Samson S, Giacomini E, Rougetet R, HertzPannier L, Le Bihan D, Marsault C, Baulac M (2000) Functional MR evaluation of temporal and frontal language dominance compared with the Wada test. Neurology 54:1625-1633.

Mandoki MW, Sumner GS, Hoffman RP, Riconda DL (1991) A review of Klinefelter's syndrome in children and adolescents. J Am Acad Child Adolesc Psychiatry 30:167-172.

Marshall WA, Tanner JM (1970) Variations in the pattern of pubertal changes in boys. Arch Dis Child 45:13-23.

Matsumoto R, Nair DR, LaPresto E, Najm I, Bingaman W, Shibasaki H, Lüders HO (2004) Functional connectivity in the human language system: a cortico-cortical evoked potential study. Brain 127:2316-2330.

Patwardhan AJ, Eliez S, Bender B, Linden MG, Reiss AL (2000) Brain morphology in Klinefelter syndrome: extra $\mathrm{X}$ chromosome and testosterone supplementation. Neurology 54:2218-2223.

Patwardhan AJ, Brown WE, Bender BG, Linden MG, Eliez S, Reiss AL (2002) Reduced size of the amygdala in individuals with 47,XXY and 47,XXX karyotypes. Am J Med Genet 114:93-98.

Penfield W, Boldrey E (1937) Somatic motor and sensory representation in the cerebral cortex of man as studied by electrical stimulation. Brain 60:389-443.

Puce A, Allison T, Bentin S, Gore JC, McCarthy G (1998) Temporal cortex activation in humans viewing eye and mouth movements. J Neurosci 18:2188-2199.
Rae C, Joy P, Harasty J, Kemp A, Kuan S, Christodoulou J, Cowell CT, Coltheart M (2004) Enlarged temporal lobes in Turner syndrome: an X-chromosome effect? Cereb Cortex 14:156-164.

Ratcliffe SG, Bancroft J, Axworthy D, McLaren W (1982) Klinefelter's syndrome in adolescence. Arch Dis Child 57:6-12.

Redcay E, Dodell-Feder D, Pearrow MJ, Mavros PL, Kleiner M, Gabrieli JD, Saxe R (2010) Live face-to-face interaction during fMRI: a new tool for social cognitive neuroscience. Neuroimage 50:1639-1647.

Reiss AL, Eliez S, Schmitt JE, Patwardhan A, Haberecht M (2000) Brain imaging in neurogenetic conditions: realizing the potential of behavioral neurogenetics research. Ment Retard Dev Disabil Res Rev 6:186-197.

Rezaie R, Daly EM, Cutter WJ, Murphy DG, Robertson DM, DeLisi LE, Mackay CE, Barrick TR, Crow TJ, Roberts N (2009) The influence of sex chromosome aneuploidy on brain asymmetry. Am J Med Genet B Neuropsychiatr Genet 150B:74-85.

Rose AB, Merke DP, Clasen LS, Rosenthal MA, Wallace GL, Vaituzis AC, Fields JD, Giedd JN (2004) Effects of hormones and sex chromosomes on stress-influenced regions of the developing pediatric brain. Ann N Y Acad Sci 1032:231-233.

Ross JL, Samango-Sprouse C, Lahlou N, Kowal K, Elder FF, Zinn A (2005) Early androgen deficiency in infants and young boys with 47,XXY Klinefelter syndrome. Horm Res 64:39-45.

Ross JL, Roeltgen DP, Stefanatos G, Benecke R, Zeger MP, Kushner H, Ramos P, Elder FF, Zinn AR (2008) Cognitive and motor development during childhood in boys with Klinefelter syndrome. Am J Med Genet A 146A:708-719.

Shen D, Liu D, Liu H, Clasen L, Giedd J, Davatzikos C (2004) Automated morphometric study of brain variation in XXY males. Neuroimage 23:648-653.

Simerly RB, Chang C, Muramatsu M, Swanson LW (1990) Distribution of androgen and estrogen receptor mRNA-containing cells in the rat brain: an in situ hybridization study. J Comp Neurol 294:76-95.

Steinman K, Ross J, Lai S, Reiss A, Hoeft F (2009) Structural and functional neuroimaging in Klinefelter (47,XXY) syndrome: a review of the literature and preliminary results from a functional magnetic resonance imaging study of language. Dev Disabil Res Rev 15:295-308.

Takeuchi H, Sekiguchi A, Taki Y, Yokoyama S, Yomogida Y, Komuro N, Yamanouchi T, Suzuki S, Kawashima R (2010) Training of working memory impacts structural connectivity. J Neurosci 30:3297-3303.

Talairach J, Tornoux P (1988) Co-planar stereotaxic atlas of the human brain: 3-dimensional proportional system: an approach to cerebral imaging. Stuttgart: Georg Thieme.

Topper E, Dickerman Z, Prager-Lewin R, Kaufman H, Maimon Z, Laron Z (1982) Puberty in 24 patients with Klinefelter syndrome. Eur J Pediatr $139: 8-12$.

van Rijn S, Swaab H, Aleman A, Kahn RS (2006) X chromosomal effects on social cognitive processing and emotion regulation: a study with Klinefelter men (47,XXY). Schizophr Res 84:194-203.

van Rijn S, Aleman A, Swaab H, Vink M, Sommer I, Kahn RS (2008) Effects of an extra X chromosome on language lateralization: an fMRI study with Klinefelter men (47,XXY). Schizophr Res 101:17-25.

Vawter MP, Harvey PD, DeLisi LE (2007) Dysregulation of X-linked gene expression in Klinefelter's syndrome and association with verbal cognition. Am J Med Genet B Neuropsychiatr Genet 144B:728-734.

Warwick MM, Doody GA, Lawrie SM, Kestelman JN, Best JJ, Johnstone EC (1999) Volumetric magnetic resonance imaging study of the brain in subjects with sex chromosome aneuploidies. J Neurol Neurosurg Psychiatry 66:628-632.

Wattendorf DJ, Muenke M (2005) Klinefelter syndrome. Am Fam Physician 72:2259-2262.

Wise RJ, Greene J, Büchel C, Scott SK (1999) Brain regions involved in articulation. Lancet 353:1057-1061. 